\title{
Optimized cell geometry for buffer-gas-cooled molecular-beam sources
}

\author{
Vijay Singh, ${ }^{1,2}$ Amit K. Samanta, ${ }^{1}$ Nils Roth,,${ }^{1,3}$ Daniel Gusa, ${ }^{1}$ Tim Ossenbrüggen,,${ }^{1}$ Igor Rubinsky, ${ }^{1,2}$ \\ Daniel A. Horke, ${ }^{1,2}$ and Jochen Küpper ${ }^{1,2,3, *}$ \\ ${ }^{1}$ Center for Free-Electron Laser Science, Deutsches Elektronen-Synchrotron DESY, Notkestrasse 85, 22607 Hamburg, Germany \\ ${ }^{2}$ The Hamburg Center for Ultrafast Imaging, Universität Hamburg, Luruper Chaussee 149, 22761 Hamburg, Germany \\ ${ }^{3}$ Department of Physics, Universität Hamburg, Luruper Chaussee 149, 22761 Hamburg, Germany
}

(Received 1 February 2018; published 21 March 2018)

\begin{abstract}
We have designed, constructed, and commissioned a cryogenic helium buffer-gas source for producing a cryogenically cooled molecular beam and evaluated the effect of different cell geometries on the intensity of the produced molecular beam, using ammonia as a test molecule. Planar and conical entrance and exit geometries are tested. We observe a threefold enhancement in the $\mathrm{NH}_{3}$ signal for a cell with planar entrance and conicalexit geometry, compared to that for a typically used "boxlike" geometry with planar entrance and exit. These observations are rationalized by flow field simulations for the different buffer-gas cell geometries. The full thermalization of molecules with the helium buffer gas is confirmed through rotationally resolved resonanceenhanced multiphoton ionization spectra yielding a rotational temperature of $5 \mathrm{~K}$.
\end{abstract}

DOI: 10.1103/PhysRevA.97.032704

\section{INTRODUCTION}

The production of slow and cold molecular beams with high flux is a prerequisite for many modern experiments, such as the study of cold reactive collisions [1-3] or Zeeman and Stark deceleration [4,5]. Furthermore, they are the starting point for further cooling of molecules to ultracold temperatures via evaporative or sympathetic cooling with ultracold atoms [6,7] or by direct laser cooling of molecules [8-10]. These novel sources of ultracold molecular systems enable ultrahigh precision measurements, such as the investigation of spatial and temporal variations of fundamental constants [11,12], and experimental searches of the electric dipole moment of the electron $[13,14]$.

The advent of ultrashort $\mathrm{x}$-ray free-electron lasers has now sparked an immense interest in producing cold beams of large molecules and particles for x-ray diffractive imaging with atomic resolution [15-19]. These experiments require pure samples delivered into a micrometer-sized interaction region, which can be achieved using electrostatic control techniques applied to cold molecular beams [19-21]. Moreover, these techniques also allow the dispersion of rotational states, such that the coldest molecules from a molecular beam can be selected, yielding, e.g., higher degrees of molecular one- and three-dimensional alignment and orientation [22-27].

All the aforementioned experiments, from laboratory-based high-precision spectroscopy to facility-based novel imaging methods, require stable sources producing a high flux of cold molecules in the gas phase. One approach to achieve this is the use of buffer-gas cooling techniques [28,29], in which a molecular sample is cooled through collisions with a cryogenic coolant, typically helium. In this paper we report on

\footnotetext{
*jochen.kuepper@cfel.de; https://www.controlled-molecule-imaging.org
}

our cryogenic buffer-gas-cell source, including the geometric optimization of the cell shape to avoid molecular losses within the cell and produce a higher-flux molecular beam.

To the best of our knowledge, this represents the first study optimizing the cryogenic cell shape for the production of higher-flux molecular beams. Our experimental measurement together with detailed flow field simulations suggest that a cryogenic cell with a planar entrance and exit, i.e., the typical "boxlike" geometry, produces vortices in the flow fields, resulting in trapping of molecules, and eventually loss of molecular density by diffusion to the cold cell walls. We find that this can be avoided, and a three times higher molecular flux achieved, through the use of a conical-exit aperture. By using ammonia as a test system we confirm that the altered geometry does not affect the thermalization of molecules, which emerge from the cell with a rotational temperature of $\sim 5 \mathrm{~K}$.

\section{EXPERIMENTAL METHODS}

A schematic diagram of our molecular beam apparatus is shown in Fig. 1(a). It primarily consists of a buffer-gascell source cooled by a pulsed-tube refrigerator (Sumitomo RP082E2) and a time-of-flight mass spectrometer (TOF-MS) with a multichannel plate (MCP) detector. These are mounted in vacuum chambers evacuated using turbomolecular pumps to typical operating pressures of $2 \times 10^{-6}$ mbar (source region, Pfeiffer Vacuum HiPace 2300) and $8 \times 10^{-7}$ mbar (detection region, Pfeiffer Vacuum HiPace 300).

The cryocooler contains two cooling stages, cooled to base temperatures of 28 and $3.4 \mathrm{~K}$, respectively. Each cooling stage is encased by a radiation shield, not shown in Fig. 1(a), to avoid unwanted heat load from blackbody radiation. The outer radiation shield is made of aluminum and thermally connected to the first stage of the cryocooler. The inner radiation shield is made of oxygen-free copper and thermally connected to the second stage. The inside of this shield is furthermore covered 


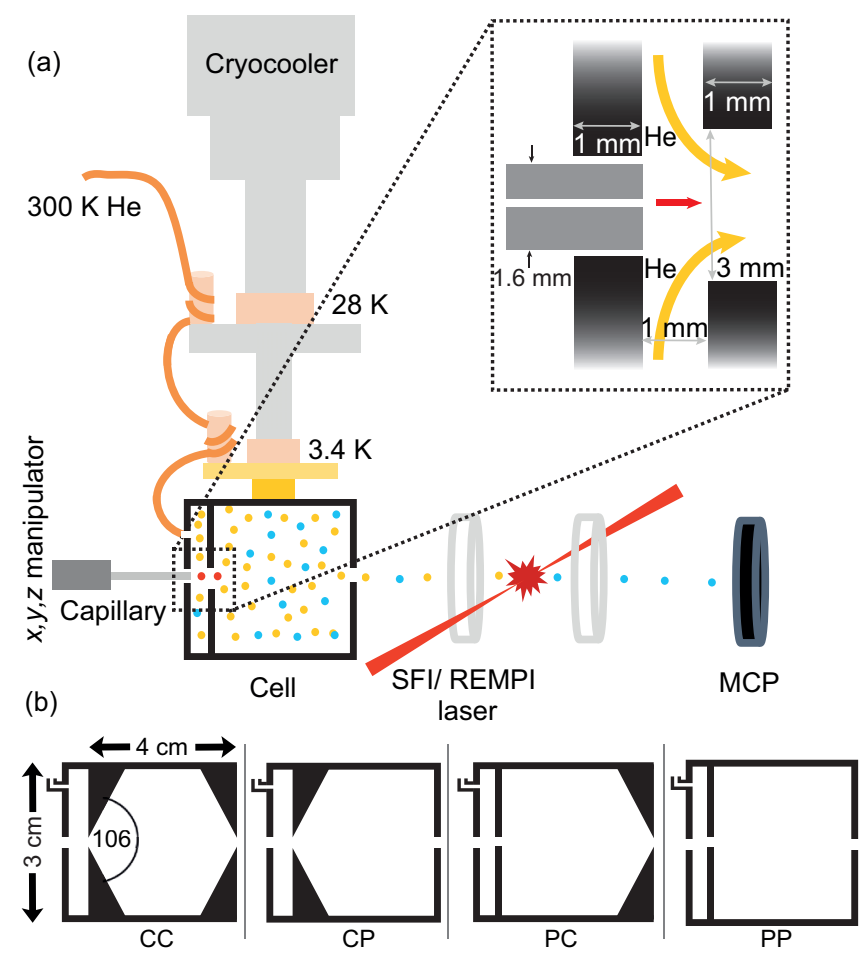

FIG. 1. (a) Schematic of the cryogenic buffer-gas-cell setup. A two-stage cryocooler holds a copper cell, cooled to $3.4 \mathrm{~K}$ base temperature. The cell has a small volume at the front where cold helium (orange dots) is introduced, which then flows radially into the main thermalization cell. Warm molecules (red dots) are injected from a heated stainless steel capillary, carried by the radial helium flow, and eventually thermalize to the buffer-gas temperature (blue dots) via collisions. The produced molecular beam is detected in a time-of-flight mass spectrometer; see text for details. (b) The buffer-gas cell has detachable entrance and exit end caps, which can either be planar, with a $180^{\circ}$ opening angle, or conical, with a $106^{\circ}$ opening angle, resulting in four distinct geometries.

with coconut charcoal acting as a cryogenic sorption pump when cooled below $10 \mathrm{~K}$ [30]. Both radiation shields contain $1 \mathrm{~cm}$ diameter apertures at the entrance and exit sides to allow introduction and extraction of sample from the buffer-gas cell; see below. The buffer-gas cell itself is attached to the second stage of the cryocooler and cooled down to $3.4 \mathrm{~K}$ base temperature. The temperatures at the first and second cooling stages and at the buffer-gas cell are continuously monitored using calibrated silicon diode temperature sensors (DT-670, Lake Shore Cryotronics).

The main body of the buffer-gas cell is a hollow copper cylinder of diameter $3 \mathrm{~cm}$ and length $2 \mathrm{~cm}$. Attach to this are exchangeable copper end caps at the entrance and exit sides, each $1 \mathrm{~cm}$ deep and with entrance and exit aperture diameters of 3 and $2 \mathrm{~mm}$, respectively. In this study we test two different end-cap geometries for the entrance and exit sides of the cell: a common planar end cap with a $180^{\circ}$ opening angle, and a conical end cap with an opening angle of $106^{\circ}$, as indicated in Fig. 1(b). This leads to four different geometries of the buffergas cell, depending on the combination of entrance and exit end cap: conical-conical (CC), conical-planar (CP), planar-conical (PC), and planar-planar (PP).
Helium buffer gas is introduced into the cell from the same side as the molecular sample [left-hand side in Fig. 1(a)] using a $1.64 \mathrm{~mm}$ inner diameter (ID) copper tube. This is thermally anchored using copper bobbins attached to both cooling stages to cool the helium to $3.4 \mathrm{~K}$ before it enters the cell. The helium is introduced into a small volume before the actual buffer-gas cell, which it rapidly fills and then provides a radial helium flow into the cell through the $3 \mathrm{~mm}$ entrance end cap aperture; see inset in Fig. 1(a). The exact flow of helium into the cell is controlled using a digital flow meter (Vögtlin Instruments GSC-A9TA-BB21) calibrated for helium and situated outside vacuum. The warm molecular sample, $\mathrm{NH}_{3}$ with purity $>99.99 \%$ and kept at 6 mbar pressure, is introduced into the buffer-gas cell using a $10 \mathrm{~cm}$ long stainless steel capillary with an inside diameter of $254 \mu \mathrm{m}$. This capillary is connected to the sample delivery manifold by $6 \mathrm{~mm}$ stainless steel tubing and the complete assembly is attached to the vacuum chamber using a three-dimensional $(x, y, z)$ position manipulator. This allows precise manipulation of the capillary in and out of the buffer-gas cell through the radiation shields and cell-entrance apertures. During experiments the capillary tip is located around $1 \mathrm{~mm}$ inside the cell-entrance aperture, as indicated in Fig. 1(a). Molecules are then carried into the cell by the flow of cold helium. To prevent freezing of sample inside the capillary, it is heated to $80^{\circ} \mathrm{C}$ using resistive heating wire and the temperature is continuously monitored with a thermocouple. For $\mathrm{NH}_{3}$ pressures of $\leqslant 6$ mbar this prevents capillary clogging and the experiment runs continuously for an entire day, while for $\mathrm{NH}_{3}$ pressure of $\geqslant 10$ mbar clogging occurs after around 3 hours for the used capillary position. Within the cell warm molecules collisionally thermalize with the cold helium, before being extracted through an exit aperture of $2 \mathrm{~mm}$ diameter into high vacuum. The formed molecular beam passes through the apertures in both radiation shields and enters into the ion optics of the TOF-MS, located $51 \mathrm{~mm}$ downstream of the buffer-gas-cell exit.

We can estimate the approximate density $n_{\mathrm{He}}$ of helium buffer gas in the cell by considering a steady state with a constant and controllable helium flow into the cell, $f$, and a corresponding flow through the exit aperture with area $A_{\text {aperture }}$,

$$
n_{\mathrm{He}}=\frac{4 f}{A_{\text {aperture }} \bar{v}} .
$$

Here $\bar{v}$ is the mean thermal velocity of helium buffer gas near the exit aperture, which can be evaluated as

$$
\bar{v}=\sqrt{\frac{8 k_{\mathrm{B}} T}{\pi m},}
$$

where $k_{\mathrm{B}}$ is the Boltzmann constant, $T_{\mathrm{He}}$ the buffer-gas temperature, and $m_{\mathrm{He}}$ the mass of the buffer gas. For a typical flow of $10 \mathrm{ml} / \mathrm{min}^{1}$ into the buffer-gas cell and an exit aperture of $2 \mathrm{~mm}$; this corresponds to a density of $4 \times 10^{16} \mathrm{~cm}^{-3}$ inside the cell. We furthermore simulate the helium flow field inside the buffer-gas cell using a finite-element solver,

\footnotetext{
${ }^{1}$ We use "milliliter normal per minute" as volume-equivalent of mass flow, with standard temperature and pressure conditions of $0{ }^{\circ} \mathrm{C}$ and $1.013 \mathrm{bar}$; this is identical to the often used literal specification "sccm."
} 


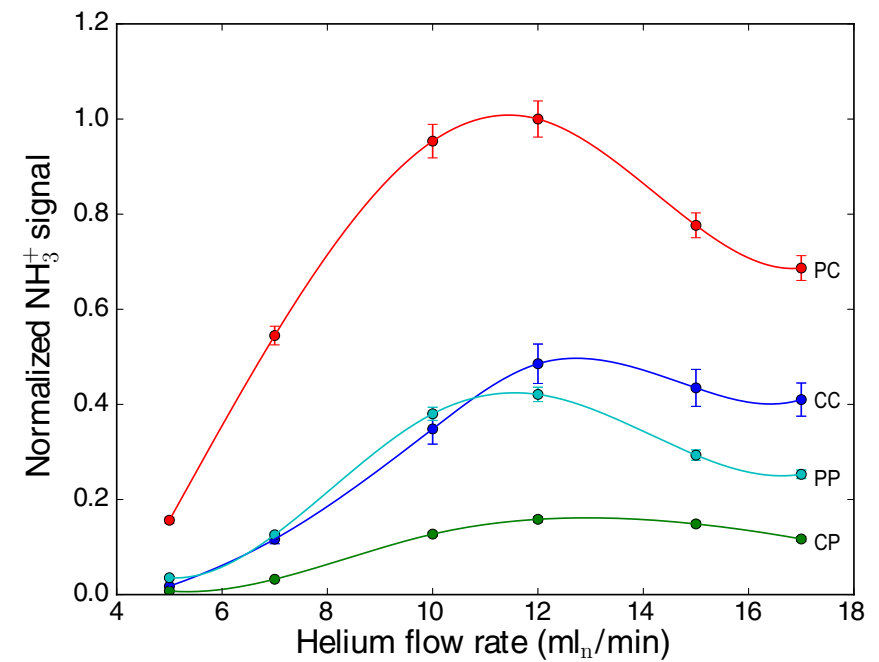

FIG. 2. Measured ammonia-ion signal (solid circles), from SFI of ammonia, as a function of helium flow rate for the four geometries of the cryogenic cell; the signal decreases from PC over CC and PP, to CP. Lines are third-order polynomial fits to guide the eye. All geometries show an initial linear increase, until a maximum is reached around 12 $\mathrm{ml}_{\mathrm{n}} /$ min helium flow. The cell geometry significantly affects measured signal intensity, with a planar-conical cell providing the most intense molecular beam.

Cosmol MulTiPhysics with laminar flow interface [31], treating helium as an ideal gas at $4 \mathrm{~K}$ temperature.

Between the ion optics of the TOF-MS spectrometer, molecules are ionized by a pulsed laser system. To monitor the total molecular flux we utilize strong-field ionization (SFI) by an amplified Ti:sapphire laser (Spectra Physics Spitfire Ace) yielding $40 \mathrm{fs}$ pulses at $1 \mathrm{kHz}$ repetition rate, focused to typical field strengths of $10^{13} \mathrm{~W} / \mathrm{cm}^{2}$ in a $100 \mu \mathrm{m}$ spot (FWHM) by a $f=750 \mathrm{~mm}$ plano-convex lens. For rotational-state selective detection we use resonance-enhanced multiphoton ionization (REMPI) of ammonia via the $\tilde{C} \leftarrow \tilde{X}$ transition at 63846$63919 \mathrm{~cm}^{-1}$ [32]. Narrowband laser pulses are provided by a tunable dye laser (FineAdjustment) using DCM dye, pumped by the frequency doubled output of a Nd:YAG laser (InnoLas). Typical pulse energies are $2 \mathrm{~mJ}$ in a $100 \mu \mathrm{m}$ spot (FWHM) at $20 \mathrm{~Hz}$ repetition rate. Produced ions are subsequently detected using a time-of-flight mass spectrometer with a typical mass resolution $m / \Delta m \approx 1000$.

\section{RESULTS AND DISCUSSION}

In Fig. 2 we show the integrated $\mathrm{NH}_{3}^{+}$signal for the four different buffer-gas-cell geometries, following strongfield ionization, as a function of the helium flow rate into the cell. Regardless of the cell geometry, we observe the same general trend: the ammonia signal first increases approximately linearly with helium flow rate until it reaches a maximum at around $10-12 \mathrm{ml}_{\mathrm{n}} / \mathrm{min}$ of helium, after which the signal plateaus off or decreases slowly.

The initial near-linear rise of the signal with increasing helium flow can be rationalized with the more efficient extraction of molecules from the buffer-gas cell. This extraction efficiency is often expressed in terms of the cell-extraction parameter, $\gamma_{\mathrm{e}}$,

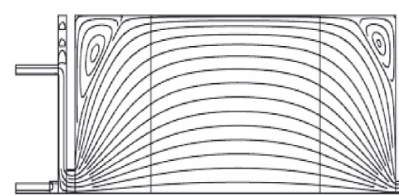

(a) planar-planar

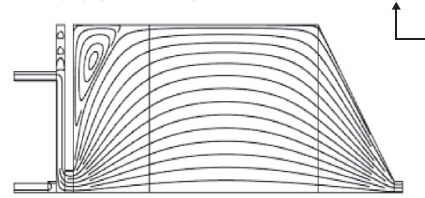

(c) planar-conical

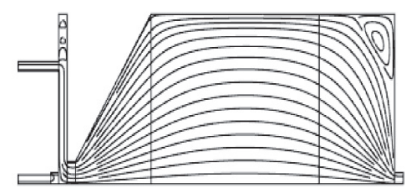

(b) conical-planar

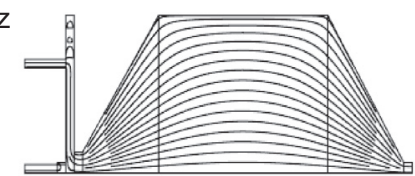

(d) conical-conical
FIG. 3. Simulations of the helium flow field for four different geometries of our cryogenic cell. Depicted are streamlines with the helium flowing from left to right. For clarity, only one half of the cylindrically symmetric $r, z$ plane is shown.

defined as the ratio of the diffusion time $\tau_{\text {diff }}$ and the typical cell pump-out time $\tau_{\text {pump }}$ [28]:

$$
\gamma_{\mathrm{e}}=\frac{\tau_{\text {diff }}}{\tau_{\text {pump }}}=\frac{4}{9 \pi} \frac{n_{\mathrm{He}} \sigma A_{\text {aperture }}}{l_{\text {cell }}} \approx \frac{\sigma f}{l_{\text {cell }} \bar{v}} .
$$

Here $\sigma$ is the elastic collision cross section for moleculehelium collisions, $l_{\text {cell }}$ the cell length, and $f$ is the controllable helium flow into the cell. For $\gamma_{\mathrm{e}} \leqslant 1$ extraction from the cell is diffusion limited, and increased helium flow leads to a corresponding increase in extraction efficiency, and hence higher signals. For the case where $\gamma_{\mathrm{e}} \geqslant 1$, however, most molecules are extracted from the cell before diffusing to the cell walls, in a regime termed hydrodynamic enhancement. Once this regime is reached an increase in helium flow rate does not have a significant influence on the extraction efficiency anymore. For our cell, an extraction parameter of $\gamma_{\mathrm{e}} \approx 1$ is expected at around $13 \mathrm{ml}_{\mathrm{n}} / \mathrm{min}$ flow rate, assuming a collision cross section of $\sigma=10^{-14} \mathrm{~cm}^{2}$ [28]. This correlates well with the observed signal emerging from the buffer-gas cell: For all geometries, we first observe a near-linear increase with helium flow rate, corresponding to the diffusion-limited case, until the flow rate reaches around $10-12 \mathrm{ml}_{\mathrm{n}} / \mathrm{min}$. Beyond this we enter the hydrodynamic entrainment regime, where the signal plateaus off or decreases slowly $[28,33]^{2}$

While the general trend between the observed ammonia signal and the applied helium flow is similar for all cell geometries tested, we do observe marked differences in the absolute signal levels obtained for different cell configurations, as shown in Fig. 2. The cell with planar entrance and conicalexit end caps (PC) produces the largest signal, with about a threefold increase compared to the regular planar-planar (PP) shape. In contrast to this, the cell with conical-entrance and planar-exit end caps $(\mathrm{CP})$ produces the smallest signal levels, almost tenfold smaller than the PC configuration.

\footnotetext{
${ }^{2}$ One possible loss mechanism of $\mathrm{NH}_{3}$ signal is the formation of dimers or clusters via molecule-molecule collisions near the cell entrance. However, our $\mathrm{NH}_{3}$ signal is observed to increase linearly with $\mathrm{NH}_{3}$ inlet pressure and we rule out the formation of dimers or clusters and hence $\mathrm{NH}_{3}$ loss by this mechanism.
} 
TABLE I. Simulated pressures and helium flows within the cell for the four geometries at $10 \mathrm{ml}_{\mathrm{n}} / \mathrm{min}$ helium flow rate.

\begin{tabular}{lcccc}
\hline \hline Geometry & $\begin{array}{c}\text { Pressure } \\
(\mathrm{mbar})\end{array}$ & $\begin{array}{c}\text { Density } \\
\left(\mathrm{cm}^{-3}\right)\end{array}$ & $\begin{array}{c}\text { Outlet flow } \\
\left(\mathrm{ml}_{\mathrm{n}} / \mathrm{min}\right)\end{array}$ & $\begin{array}{c}\text { Inlet flow } \\
\left(\mathrm{ml}_{\mathrm{n}} / \mathrm{min}\right)\end{array}$ \\
\hline $\mathrm{CC}$ & 0.033 & $7.9 \times 10^{16}$ & 9.6 & 0.4 \\
$\mathrm{CP}$ & 0.048 & $1.2 \times 10^{17}$ & 9.2 & 0.8 \\
$\mathrm{PC}$ & 0.032 & $7.7 \times 10^{16}$ & 9.6 & 0.4 \\
$\mathrm{PP}$ & 0.048 & $1.2 \times 10^{17}$ & 9.2 & 0.8 \\
\hline \hline
\end{tabular}

In order to qualitatively understand the observed differences for the four cell geometries, we have simulated the helium flow fields in the cryogenic cell (see Sec.II for details) at $10 \mathrm{ml} / \mathrm{min}$ of helium flow and a pressure of $10^{-6}$ mbar at the cell exit. The resulting flow fields are shown in Fig. 3 as slices through the cylindrically-symmetric cell volume, i.e., in $r, z$ space, and we further extract the helium pressures inside the cell as well as the flow of helium through the entrance and exit of the cell, shown in Table I.

These flows and cell pressures clearly show that the helium environment within the cell depends on the geometry of the exit end cap. A planar-exit end cap leads to reduced transmission through the exit aperture compared to a conical end cap, yielding a correspondingly higher pressure within the cell and loss of helium density by "back-flow" through the cell-entrance aperture. Furthermore, the simulations show that planar end caps produce vortices in the flow fields at the cell corners. This can be circumvented by the use of conical end caps, which result in an overall laminar flow. Hot ammonia molecules introduced into the cell will be thermalized with the buffer gas before reaching the exit end cap and hence follow the streamlines. The presence of vortices in the flow field can, therefore, lead to trapping of ammonia and eventually loss to the cell walls. Thus, the laminar flow produced by a conical end cap at the cell exit is clearly advantageous in avoiding diffusion loss and, therefore, entraining more $\mathrm{NH}_{3}$ molecules into the molecular beam.

Furthermore, according to our calculations the effect of the end caps on the forward velocity of the generated beams is small, but when replacing the planar-exit end cap with the conical-exit end cap it decreases from 80 to $70 \mathrm{~m} / \mathrm{s}$, which is advantageous for slow-beam and trapping experiments.

The simulation results for the extracted helium flow and cell pressures for CC and PC cell geometries are very similar, indicating that the vortices produced by the planar end cap at the cell entrance do not have any adverse influence on the helium flow through the cell. This might lead one to expect very similar ammonia signal levels for these two cell geometries. However, the measured signal levels differ by around a factor of 3. We attribute this difference to increased diffusion and loss of ammonia molecules at the cold cell walls for the conicalentrance cap due to the closer proximity of the copper walls to the inlet capillary. At the cell entrance molecules are not fully thermalized with the helium buffer gas and will not follow the helium flow field precisely. Therefore, diffusive motion is still considerable and the larger opening angle of the planar entrance cap leads to a larger distance to the cold cell walls

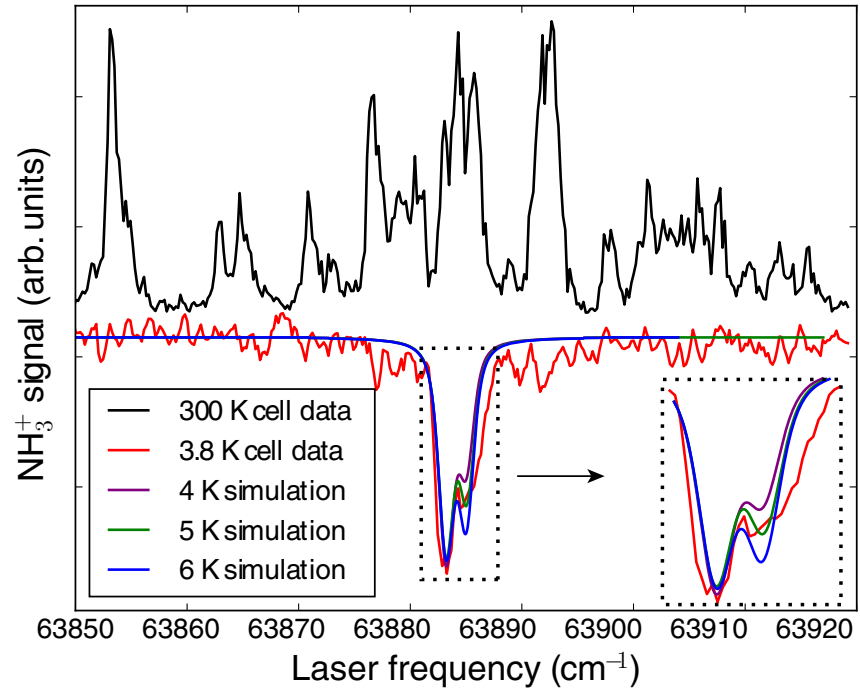

FIG. 4. REMPI spectrum of $\mathrm{NH}_{3}$ measured using a planar-conical cell at room temperature (black, upper line) and cooled to $3.8 \mathrm{~K}$ (red, lower line), together with corresponding PGOPHER simulations at 4,5 , and $6 \mathrm{~K}$ rotational temperature. In the inset, the different simulations can be distinguished on the right peak, which is strongest for $6 \mathrm{~K}$, intermediate for $5 \mathrm{~K}$, and weakest for $4 \mathrm{~K}$. The $5 \mathrm{~K}$ simulation reproduces the experimental data very well, indicating full thermalization of the molecules with the buffer gas.

and hence an increased molecular flux out of the buffer-gas cell compared to the conical-entrance geometry.

Based on these arguments and our flow field simulations, we summarize our observation as follows: at the entrance molecules are primarily lost through diffusion, making planar end caps preferential as they maximize the distance to the cold cell walls. At the cell exit molecules are thermalized and follow the helium flow field such that vortices should be avoided, which can be achieved with conical end caps. For our cell, these two effects appear to be of roughly the same magnitude, such that the planar-planar (PP) and conicalconical (CC) geometries show approximately the same signal intensity. To confirm that molecules ejected from the cell are fully thermalized with the buffer gas, we collected $2+1$ resonance-enhanced multiphoton ionization (REMPI) spectra of ammonia via the $\tilde{C}$ state. Here, an $\mathrm{NH}_{3}$ pressure of 6 mbar and a helium flow rate of $10 \mathrm{ml}_{\mathrm{n}} / \mathrm{min}$ were used, corresponding to a Reynolds number of $\sim 56$ and thus producing a molecular beam in the partially hydrodynamic regime, i.e., without any significant further acceleration or cooling when exiting the cell [28]. In Fig. 4 the spectrum is shown for both room temperature and buffer-gas cooled $\mathrm{NH}_{3}$. The strong temperature dependence of the rovibronic transitions allows us to extract precise information about the rotational temperature of ammonia. We simulated spectra using the PGOPHER software [34], with literature values used for the rotational constants in the electronic ground $(\tilde{X})$ and excited $(\tilde{C})$ states [32]. The simulated spectrum for a rotational temperature of $5 \mathrm{~K}$ is shown as a solid green line in Fig. 4. It matches the experimental data for buffer-gas cooled $\mathrm{NH}_{3}$ very well. As the intensities of the two observable transitions $(J=0,1)$ are very sensitive to the rotational temperature, we also simulated spectra at 4 and $6 \mathrm{~K}$ 
(purple and blue lines in Fig. 4, respectively). These do not fit the experimental spectrum well and confirm our temperature assignment of $5 \mathrm{~K}$. This indicates complete thermalization of the molecular rotations with the helium buffer gas. Since the elastic collision cross section of the molecules with the helium is expected to be significantly higher than that for the rotational relaxation [28], the translational temperature of the molecules should also be thermalized completely. Measurements were conducted for all four cell geometries and similar rotational temperatures extracted in all cases.

\section{CONCLUSION}

In conclusion, we designed, constructed, and commissioned a cryogenic apparatus for producing helium buffer-gas cooled molecular beams. We evaluated the effect of the cell geometry on the produced molecular beam flux and observed that a cryogenic cell with a planar entrance and conical-exit end caps produces around three times more flux compared to the typically used "boxlike" geometry with planar entrance and exit. This is attributed to the smaller diffusion loss at the entrance due to the larger opening angle of a planar end cap and an optimum transmission at the exit resulting from purely laminar helium flow without vortices for the conical end cap. We confirmed the full thermalization of molecules with the buffer gas through REMPI measurements that show a rotational temperature of $5 \mathrm{~K}$ for all cell geometries.

We showed that a simple geometric optimization of the cell shape can significantly improve the molecular beam flux, and we are currently expanding this technique to larger molecules and nanoparticles. Such a stable, cold, and high-flux beam of molecules will be beneficial to a wide range of molecular physics experiments, including novel single-particle $x$-ray diffractive imaging approaches.

\section{ACKNOWLEDGMENTS}

In addition to DESY, this work has been supported by the European Research Council under the European Union's Seventh Framework Programme (FP7/2007-2013) through the Consolidator Grant COMOTION (ERC-614507-Küpper), by the excellence cluster "The Hamburg Center for Ultrafast Imaging - Structure, Dynamics and Control of Matter at the Atomic Scale" of the Deutsche Forschungsgemeinschaft (CUI, DFG-EXC1074), and by the Helmholtz Gemeinschaft through the "Impuls- und Vernetzungsfond."
[1] A. B. Henson, S. Gersten, Y. Shagam, J. Narevicius, and E. Narevicius, Observation of resonances in Penning ionization reactions at sub-Kelvin temperatures in merged beams, Science 338, 234 (2012).

[2] V. Singh, K. S. Hardman, N. Tariq, M.-J. Lu, A. Ellis, M. J. Morrison, and J. D. Weinstein, Chemical Reactions of Atomic Lithium and Molecular Calcium Monohydride at $1 \mathrm{~K}$, Phys. Rev. Lett. 108, 203201 (2012).

[3] Y.-P. Chang, K. Długołęcki, J. Küpper, D. Rösch, D. Wild, and S. Willitsch, Specific chemical reactivities of spatially separated 3-aminophenol conformers with cold $\mathrm{Ca}^{+}$ions, Science 342, 98 (2013).

[4] N. Vanhaecke, U. Meier, M. Andrist, B. H. Meier, and F. Merkt, Multistage Zeeman deceleration of hydrogen atoms, Phys. Rev. A 75, 031402(R) (2007).

[5] H. L. Bethlem, G. Berden, and G. Meijer, Decelerating Neutral Dipolar Molecules, Phys. Rev. Lett. 83, 1558 (1999).

[6] B. K. Stuhl, M. T. Hummon, M. Yeo, G. Quéméner, J. L. Bohn, and J. Ye, Evaporative cooling of the dipolar hydroxyl radical, Nature (London) 492, 396 (2012).

[7] J. Lim, M. D. Frye, J. M. Hutson, and M. R. Tarbutt, Modeling sympathetic cooling of molecules by ultracold atoms, Phys. Rev. A 92, 053419 (2015).

[8] E. S. Shuman, J. F. Barry, and D. DeMille, Laser cooling of a diatomic molecule, Nature (London) 467, 820 (2010).

[9] V. Zhelyazkova, A. Cournol, T. E. Wall, A. Matsushima, J. J. Hudson, E. A. Hinds, M. R. Tarbutt, and B. E. Sauer, Laser cooling and slowing of CaF molecules, Phys. Rev. A 89, 053416 (2014).

[10] I. Kozyryev, L. Baum, K. Matsuda, B. L. Augenbraun, L. Anderegg, A. P. Sedlack, and J. M. Doyle, Sisyphus Laser Cooling of a Polyatomic Molecule, Phys. Rev. Lett. 118, 173201 (2017).
[11] L. Santamaria, V. D. Sarno, I. Ricciardi, S. Mosca, M. D. Rosa, G. Santambrogio, P. Maddaloni, and P. D. Natale, Assessing the time constancy of the proton-to-electron mass ratio by precision ro-vibrational spectroscopy of a cold molecular beam, J. Mol. Spectrosc. 300, 116 (2014).

[12] S. Truppe, R. J. Hendricks, S. K. Tokunaga, H. J. Lewandowski, M. G. Kozlov, C. Henkel, E. A. Hinds, and M. R. Tarbutt, A search for varying fundamental constants using hertz-level frequency measurements of cold $\mathrm{CH}$ molecules, Nat. Commun. 4, 2600 (2013).

[13] J. J. Hudson, D. M. Kara, I. J. Smallman, B. E. Sauer, M. R. Tarbutt, and E. A. Hinds, Improved measurement of the shape of the electron, Nature (London) 473, 493 (2011).

[14] The ACME Collaboration, J. Baron, W. C. Campbell, D. DeMille, J. M. Doyle, G. Gabrielse, Y. V. Gurevich, P. W. Hess, N. R. Hutzler, E. Kirilov, I. Kozyryev, B. R. O'Leary, C. D. Panda, E. S. Petrik, B. Spaun, A. C. Vutha, and A. D. West, Order of magnitude smaller limit on the electric dipole moment of the electron, Science 343, 269 (2014).

[15] M. M. Seibert, T. Ekeberg, F. R. N. C. Maia, M. Svenda, J. Andreasson, O. Jönsson, D. Odić, B. Iwan, A. Rocker, D. Westphal, M. Hantke, D. P Deponte, A. Barty, J. Schulz, L. Gumprecht, N. Coppola, A. Aquila, M. Liang, T. A. White, A. Martin et al., Single mimivirus particles intercepted and imaged with an x-ray laser, Nature (London) 470, 78 (2011).

[16] T. Ekeberg, M. Svenda, C. Abergel, F. R. N. C. Maia, V. Seltzer, J.-M. Claverie, M. Hantke, O. Jönsson, C. Nettelblad, G. van der Schot, M. Liang, D. P. Deponte, A. Barty, M. M. Seibert, B. Iwan, I. Andersson, N. D. Loh, A. V. Martin, H. Chapman, C. Bostedt, J. D. Bozek et al., Three-Dimensional Reconstruction of the Giant Mimivirus Particle with an X-Ray Free-Electron Laser, Phys. Rev. Lett. 114, 098102 (2015). 
[17] A. Barty, J. Küpper, and H. N. Chapman, Molecular imaging using x-ray free-electron lasers, Annu. Rev. Phys. Chem. 64, 415 (2013).

[18] J. Küpper, S. Stern, L. Holmegaard, F. Filsinger, A. Rouzée, A. Rudenko, P. Johnsson, A. V. Martin, M. Adolph, A. Aquila, S. Bajt, A. Barty, C. Bostedt, J. Bozek, C. Caleman, R. Coffee, N. Coppola, T. Delmas, S. Epp, B. Erk et al., X-Ray Diffraction from Isolated and Strongly Aligned Gas-Phase Molecules with a Free-Electron Laser, Phys. Rev. Lett. 112, 083002 (2014).

[19] F. Filsinger, G. Meijer, H. Stapelfeldt, H. Chapman, and J. Küpper, State- and conformer-selected beams of aligned and oriented molecules for ultrafast diffraction studies, Phys. Chem. Chem. Phys. 13, 2076 (2011).

[20] F. Filsinger, U. Erlekam, G. von Helden, J. Küpper, and G. Meijer, Selector for Structural Isomers of Neutral Molecules, Phys. Rev. Lett. 100, 133003 (2008).

[21] Y.-P. Chang, D. A. Horke, S. Trippel, and J. Küpper, Spatiallycontrolled complex molecules and their applications, Int. Rev. Phys. Chem. 34, 557 (2015).

[22] L. Holmegaard, J. H. Nielsen, I. Nevo, H. Stapelfeldt, F. Filsinger, J. Küpper, and G. Meijer, Laser-Induced Alignment and Orientation of Quantum-State-Selected Large Molecules, Phys. Rev. Lett. 102, 023001 (2009).

[23] F. Filsinger, J. Küpper, G. Meijer, L. Holmegaard, J. H. Nielsen, I. Nevo, J. L. Hansen, and H. Stapelfeldt, Quantum-state selection, alignment, and orientation of large molecules using static electric and laser fields, J. Chem. Phys. 131, 064309 (2009).

[24] I. Nevo, L. Holmegaard, J. H. Nielsen, J. L. Hansen, H. Stapelfeldt, F. Filsinger, G. Meijer, and J. Küpper, Laserinduced 3D alignment and orientation of quantum stateselected molecules, Phys. Chem. Chem. Phys. 11, 9912 (2009).
[25] S. Trippel, T. Mullins, N. L. M. Müller, J. S. Kienitz, K. Długołęcki, and J. Küpper, Strongly aligned and oriented molecular samples at a $\mathrm{kHz}$ repetition rate, Mol. Phys. 111, 1738 (2013).

[26] S. Trippel, T. Mullins, N. L. M. Müller, J. S. Kienitz, J. J. Omiste, H. Stapelfeldt, R. González-Férez, and J. Küpper, Strongly driven quantum pendulum of the carbonyl sulfide molecule, Phys. Rev. A 89, 051401(R) (2014).

[27] S. Trippel, T. Mullins, N. L. M. Müller, J. S. Kienitz, R. González-Férez, and J. Küpper, Two-State Wave Packet for Strong Field-Free Molecular Orientation, Phys. Rev. Lett. 114, 103003 (2015).

[28] N. R. Hutzler, H.-I. Lu, and J. M. Doyle, The buffer gas beam: An intense, cold, and slow source for atoms and molecules, Chem. Rev. 112, 4803 (2012).

[29] S. Truppe, M. Hambach, S. M. Skoff, N. E. Bulleid, J. S. Bumby, R. J. Hendricks, E. A. Hinds, B. E. Sauer, and M. R. Tarbutt, A buffer gas beam source for short, intense and slow molecular pulses, J. Mod. Opt. 65, 648 (2018).

[30] A. G. Tobin, D. W. Sedgley, T. H. Batzer, and W. R. Call, Evaluation of charcoal sorbents for helium cryopumping in fusion reactors, J. Vac. Sci. Technol. A 5, 101 (1987).

[31] COMSOL Multiphysics v. 5.3, http://www.comsol.com, COMSOL AB, Stockholm, Sweden.

[32] M. Nolde, K. M. Weitzel, and C. M. Western, The resonance enhanced multiphoton ionisation spectroscopy of ammonia isotopomers $\mathrm{NH}_{3}, \mathrm{NH}_{2} \mathrm{D}, \mathrm{NHD}_{2}$ and $\mathrm{ND}_{3}$, Phys. Chem. Chem. Phys. 7, 1527 (2005)

[33] D. Patterson and J. M. Doyle, Bright, guided molecular beam with hydrodynamic enhancement, J. Chem. Phys. 126, 154307 (2007).

[34] C. M. Western, PGOPHER, A Program for Simulating Rotational Structure, Vibrational and Electronic Spectra, University of Bristol, http://pgopher.chm.bris.ac.uk. 